\title{
Lp(a)-cholesterol is associated with HDL- cholesterol in overweight and obese African American children and is not an independent risk factor for CVD
}

\author{
Sushma Sharma ${ }^{1,2^{*}}$, Jayshree Merchant ${ }^{3}$ and Sharon E Fleming ${ }^{1,2}$
}

\begin{abstract}
Background: The role of Lipoprotein (a) cholesterol $\{L p(a)-C\} a s$ an additional and/or independent risk factor for cardiovascular disease (CVD) is not clear. We evaluated the associations between Lp(a)-C and other CVD risk factors including plasma lipoprotein concentrations and body fatness in overweight and obese African American children.

Methods: A cross-sectional analysis was carried out using data from a sample of 121 African American children aged 9-11 years with Body Mass Index (BMI)'s greater than the 85th percentile. Body height, weight and waist circumference (WC) were measured. Fasting plasma concentrations of $L p(a)-C$, Total cholesterol (TC), High density lipoprotein cholesterol (HDL-C), Very low density lipoprotein cholesterol (VLDL-C), Intermediate density lipoprotein cholesterol (IDL-C), Low density lipoprotein cholesterol (LDL-C), and Triacylglycerides (TAG) were analyzed using the vertical auto profile (VAP) cholesterol method.
\end{abstract}

Results: After adjusting for child age, gender, and pubertal status, Lp(a)-C was positively associated with both HDL$C$ and TC, and negatively associated with VLDL-C and TAG. Including BMIz and WC as additional covariates did not alter the direction of the relationships between $L p(a)-C$ and the other lipoproteins. Finally, after adjusting for the other plasma lipoproteins, Lp(a)-C remained strongly associated with HDL-C, whereas the associations of Lp(a)-C with the other lipoproteins were not significant when HDL-C was simultaneously included in the regression models.

Conclusions: $\mathrm{Lp}(\mathrm{a})-\mathrm{C}$ was positively associated with $\mathrm{HDL}-\mathrm{C}$ and this association is not influenced by other lipoprotein subclasses or by the degree of obesity. We conclude that $L p(a)$ cholesterol is not an independent risk factor for CVD in African American children.

Keywords: Lipoprotein, Lp(a), LDL, HDL, TAG, TC, BMlz, Waist circumference, Obesity, CVD, Atherosclerosis

\section{Background}

Lipoprotein (a) particles, $\mathrm{Lp}(\mathrm{a})$, were first described by Berg in 1963 [1], and are a genetic variant of low-density lipoprotein particles linked via apoB-100 to apolipoprotein(a) [2]. Since being identified, numerous studies have reported that high plasma $\mathrm{Lp}(\mathrm{a})$ concentrations are associated with atherosclerotic/thrombotic disease, as comprehensively reviewed by others [3].

\footnotetext{
* Correspondence: sushma@berkeley.edu

'Dr Robert C and Veronica Atkins Center for Weight and Health, University of California, Berkeley, CA 94720-3104, USA

Full list of author information is available at the end of the article
}

Despite these reports, there have been conflicting results from prospective studies that evaluated $L p(a)$ as an independent risk factor for cardiovascular disease (CVD). Although the majority of studies, performed in primarily Caucasian populations, have found $\mathrm{Lp}(\mathrm{a})$ concentrations to be an independent risk factor for CVD, studies that have evaluated these relationships in African Americans have been less consistent. For example, in the biracial cohort ARIC (Atherosclerosis Risk in Communities) study of 15, 800 individuals, $\mathrm{Lp}$ (a) lipoprotein levels were reported to be positively associated with CVD as well as with preclinical atherosclerosis in both 
Black and White adults $[4,5]$. In a different study, however, high $\mathrm{Lp}$ (a) concentrations were reported not to be associated with increased risk of CVD in African Americans adults [6]. When compared to other races and ethnicities, African American adults and children have been shown to have higher plasma concentrations of $\mathrm{Lp}(\mathrm{a})$. These, among other findings, have led researchers to conclude that $L p(a)$ levels are regulated by both genetic and non-genetic factors [7], suggesting that a single Lp (a) cutoff would not confer the same CVD risk in African American and White populations [8].

In the U.S., plasma lipoprotein concentrations and blood pressures have been used as predictors for CVD screening, with cutoffs based on American Heart Association guidelines [9]. Individuals with higher than recommended concentrations of Total Cholesterol (TC), Low density lipoprotein cholesterol (LDL-C), Very low density lipoprotein cholesterol (VLDL-C), Intermediate density lipoprotein cholesterol (IDL-C) or Triglycerides (TAG), and lower High density lipoprotein cholesterol (HDL-C) would be considered at risk of CVD. To determine whether $\operatorname{Lp}(\mathrm{a})$ confers additional and independent risk, several studies have investigated the relationship of $\mathrm{Lp}$ (a) to these intermediate markers of CVD risk. Lp(a) has been reported to be positively associated with TC in Turkish adults [10] and high-school adolescents [11]; and $\mathrm{Lp}(\mathrm{a})$ and LDL-C have been reported to be positively associated in White adults [12,13], Australian Aboriginal children [14] and Taiwanese boys [15].

$\mathrm{Lp}(\mathrm{a})$ has been found also to be associated with intermediate markers of CVD risk including obesity and other lipoproteins in African Americans. Lp(a) was reported to be positively associated with TC in African American women [16] and African American 12-19 year old males [17]. Also, Lp(a) and LDL-C have been reported to be positively associated in African American men [13] and women [13,16]. Some unexpected associations have been reported. For example, Giger et al. [16] reported that $\mathrm{Lp}(\mathrm{a})$ was positively associated with HDL$\mathrm{C}$ and negatively associated with TAG, VLDL-C and obesity in premenopausal African American women; and Randall et al [17] reported a negative association between Lp(a) and TAG in obese American Americans aged 12-75 years.

To the best of our knowledge, associations between Lp (a) and intermediate markers of CVD risk have not previously been reported for young African American children. Because of an epidemic increase in childhood obesity and a subsequent increase in risk for CVD, it has been recommended that children and adolescents should be screened for lipid profiles even at a young age $[18,19]$. Therefore, knowledge regarding the association between $\mathrm{Lp}(\mathrm{a})-\mathrm{C}$ and markers of CVD risk in overweight and obese African American children would provide crucial information regarding choice of markers for CVD risk determination in at risk children, and others have determined that it is essential to evaluate pediatric cohorts individually due to heterogeneity [20]. The current study represents a secondary analysis of data generated from a community-based, type 2 diabetes prevention program for inner city, overweight and obese, African American children [21]. In this analysis, we aimed to evaluate the associations of $\mathrm{Lp}(\mathrm{a})-\mathrm{C}$ with other cardiovascular disease risk factors including lipoproteins (TC, HDL-C, LDL-C, VLDL-C, TAG) and body fatness (BMIz and waist circumference) in this sample of overweight and obese, young African American children.

\section{Methods}

\section{Study participants}

A complete set of data for this cross-sectional analysis were available for a convenience sample of 121 (56 boys and 65 girls) African-American children who were part of a community-based lifestyle modification program that aimed to reduce the risk for type 2 diabetes mellitus as described more fully elsewhere [21]. Study participants were recruited by distributing pamphlets at local recreational sites and schools in inner-city Oakland, CA. Recruitment targeted children with at least one African American parent. Exclusion criteria included the following: BMI's below the 85th percentile when matched for age and gender; 8 years of age or younger; 12 years of age or older; fasting glucose $\geq 120 \mathrm{mg} / \mathrm{dl}$; any known metabolic disease; or taking medications known to affect the study outcomes. Parental informed consent was obtained from all subjects, and all protocols were approved by the institutional review boards at the University of California Berkeley and San Francisco. More than $95 \%$ of the children enrolled in this study lived in inner-city Oakland, CA.

\section{Anthropometric measurements}

Body weight and height were measured to the nearest $0.1 \mathrm{~kg}$ and $0.1 \mathrm{~cm}$ using a digital electronic scale (BWB 800, Tanita, Japan), and a portable stadiometer, respectively. Body mass index (BMI), BMI percentiles and BMI z-scores were generated using an age and gender specific CDC calculator program http://www.cdc.gov/ nccdphp/dnpa/growthcharts/resources/sas.htm. Using a plastic, non-elastic measuring tape, waist circumference (WC) was measured just above the iliac crest with the child in the standing position and hip circumference was measured at the widest point above the greater trocanthers. Measurements were taken twice and if agreement between repeats exceeded $0.4 \mathrm{~cm}$, a third measurement was taken and the mean calculated using the closest two values. 


\section{Biochemical measurements}

After a 12-h overnight fast, participants reported to the Children's Hospital and Research Center Oakland where their blood was drawn. Plasma lipids were measured by a comprehensive lipoprotein analysis performed by a commercial lab (LabCorp). Using the vertical auto profile (VAP) cholesterol method, a modified density gradient centrifugation technique, concentrations of the following components were determined on a single sample: TC, HDL-C, VLDL-C, IDL-C, LDL-C and TAG $[22,23]$.

\section{Pubertal stage assessment}

All participants were asked to report, after an overnight fast of at least $12 \mathrm{~h}$, at the Children's Hospital and Research Center in Oakland, CA for blood sample collection. Pubertal development was assessed by measurements of serum luteinizing hormones (LH) in boys, and estradiol and LH in girls. Children were classified into stages 1 through 5 using literature-derived values and with cutoffs previously reported [24].

\section{Statistical analyses}

Initially, data for 124 participants were included in the analysis. Values for several of the plasma lipoproteins were significantly skewed, and tests for non-normality remained significant even following log transformation. Using Dixon's test for outliers, data for three children were excluded to avoid unintended bias of associations in this analysis. Thus, data for 121 participants were included in the final analysis. Gender differences for anthropometric and lipoprotein characteristics were assessed using independent two-tailed t-tests following Levene's test for equality of variances. Pearson's correlations were used to determine bivariate associations. To evaluate the primary study objectives, hierarchical multiple linear regression analyses were used, with $\mathrm{Lp}(\mathrm{a})-\mathrm{C}$ as the dependent variable. Other plasma lipoproteins were included as independent variables and models were adjusted as indicated for child age, gender, pubertal stage and body fatness. Socioeconomic status of the family was evaluated as a potential covariate but was not included in the final models since it was found to have $r<0.20$ and to not be significantly related to dependent and independent variables. Statistical procedures were performed using SPSS for Windows version 18.0 PASW (SPSS, IBM Corp.). Results with $p<0.05$ were defined to be statistically significant.

\section{Results}

Plasma lipoprotein concentrations were considered to be in the normal range for many, but not all, of the children in this sample. Using reference interval cutoffs, less than $5 \%$ of the children had values outside the normal range
Table 1 Standardized reference cutoffs for plasma lipoprotein concentrations and proportion of child participants at risk of cardiovascular disease $(n=121)$

\begin{tabular}{lcc}
\hline & Reference Cutoff $^{\mathbf{1}}, \mathbf{m g} / \mathbf{d l}$ & At risk,\% of sample \\
\hline TC & $>200$ & 17.4 \\
\hline HDL-C & $\leq 40$ & 12.4 \\
\hline VLDL-C & $>30$ & 1.7 \\
\hline IDL-C & $>20$ & 3.3 \\
\hline LDL-C & $>130$ & 14.0 \\
\hline TAG & $>150$ & 1.7 \\
\hline LP(a)-C & $>10$ & 24.8 \\
\hline
\end{tabular}

${ }^{1}$ Reference cutoff intervals taken from LabCorp standards for VAP Cholesterol analyses https://www.labcorp.com/pdf/VAP_test_flyer.pdf

Abbreviations: Total cholesterol: TC, High density lipoprotein cholesterol: HDLC, Very low density lipoprotein cholesterol: VLDL-C, Intermediate density lipoprotein cholesterol: IDL-C, Low density lipoprotein cholesterol: LDL-C, Triglycerides: TAG, Lp(a) cholesterol: $\operatorname{Lp}(\mathrm{a})-\mathrm{C}$

for IDL-C, VLDL-C and TAG; $10-20 \%$ fell outside the normal range for TC, HDL-C and LDL-C; and nearly $25 \%$ fell outside the normal range for $\mathrm{Lp}(\mathrm{a})-\mathrm{C}$ (Table 1 ). Girls in this sample of overweight and obese African American children were at a more advance pubertal stage than boys, and had significantly higher body fatness assessed by BMIz and WC values (Table 2). Differences in lipoprotein concentrations did not differ by gender. When participants were divided into three BMI

Table 2 Characteristics of participating African-American boys and girls

\begin{tabular}{llll}
\hline & Boys & Girls & p-value $^{\mathbf{1}}$ \\
\hline Sample size, $n$ & 56 & 65 & \\
\hline Anthropometrics & Mean (SEM) & & \\
\hline Age (years) & $10.7(0.1)$ & $10.6(0.1)$ & 0.575 \\
\hline Pubertal stage & $2.4(0.2)$ & $3.5(0.1)$ & $<\mathbf{0 . 0 0 1}$ \\
\hline Height (cm) & $148.1(0.02)$ & $150.8(0.01)$ & 0.248 \\
\hline Weight (kg) & $62.5(2.6)$ & $69.1(2.3)$ & 0.057 \\
\hline BMl-z score & $2.0(0.07)$ & $2.2(0.05)$ & $\mathbf{0 . 0 5 0}$ \\
\hline WC (cm) & $87.0(2.1)$ & $93.0(1.8)$ & $\mathbf{0 . 0 3 2}$ \\
\hline Plasma lipoproteins, mg/dl & & & \\
\hline TC & $168.0(3.6)$ & $168.3(4.6)$ & 0.955 \\
\hline HDL-C & $56.2(1.6)$ & $52.0(1.4)$ & 0.052 \\
\hline VLDL-C & $15.9(0.6)$ & $16.1(0.4)$ & 0.736 \\
\hline IDL-C & $9.1(0.6)$ & $9.4(0.6)$ & 0.724 \\
\hline LDL-C & $95.9(3.2)$ & $100.4(4.0)$ & 0.371 \\
\hline TAG & $68.4(4.4)$ & $75.1(3.1)$ & 0.202 \\
\hline Lp(a)-C & $7.8(0.54)$ & $8.1(0.51)$ & 0.699 \\
\hline Statistical significance & & & \\
\hline
\end{tabular}

${ }^{1}$ Statistical significance of differences determined using two-tailed t-test following Levene's test for equality of variances

Abbreviations: Body mass index: BMI, Body mass index z-score: BMlz, Waist circumference: WC, Total cholesterol: TC, High density lipoprotein cholesterol: $\mathrm{HDL}-\mathrm{C}$, Very low density lipoprotein cholesterol: VLDL-C, Intermediate density lipoprotein cholesterol: IDL-C, Low density lipoprotein cholesterol: LDL-C, Triglycerides: TAG, Lp(a) cholesterol: Lp(a)-C 
Table 3 Influence of BMI percentile category on characteristics of participating children

\begin{tabular}{|c|c|c|c|c|}
\hline \multirow[t]{2}{*}{ Classification BMI percentiles } & \multirow[t]{2}{*}{ At risk of overweight $85-95$ th } & \multicolumn{3}{|c|}{ Overweight \& Obese } \\
\hline & & $95.001-99$ th & $>99$ th & $p$-value ${ }^{1}$ \\
\hline Sample size, $\mathrm{n}$ & 24 & 48 & 49 & \\
\hline Anthropometrics & Mean (SEM) & & & \\
\hline Age (years) & $10.5(0.2)$ & $10.8(0.1)$ & $10.7(0.1)$ & 0.412 \\
\hline Pubertal stage & $2.75(0.3)$ & $3.14(0.2)$ & $2.95(0.2)$ & 0.574 \\
\hline Height (cm) & $147.3(1.8)$ & $150.2(1.3)$ & $152.1(1.3)$ & 0.118 \\
\hline Weight (kg) & $46.6(1.5)$ & $61.0(1.4)$ & $80.5(2.6)$ & $<0.001$ \\
\hline BMI-z score & $1.34(0.4)$ & $2.03(0.03)$ & $2.54(0.02)$ & $<0.001$ \\
\hline WC $(\mathrm{cm})$ & $71.8(1.4)$ & $86.9(1.19)$ & $102.4(1.8)$ & $<0.001$ \\
\hline \multicolumn{5}{|l|}{ Plasma lipoproteins, mg/dl } \\
\hline$\overline{\mathrm{TC}}$ & $177.7(7.0)$ & $161.1(4.1)$ & $170.5(4.9)$ & 0.100 \\
\hline$\overline{\mathrm{HDL}-\mathrm{C}}$ & $62.2^{\mathrm{b}}(2.8)$ & $53.0^{a}(1.4)$ & $50.8^{\mathrm{a}}(1.5)$ & $<0.001$ \\
\hline VLDL-C & $15.6(0.9)$ & $15.2(0.5)$ & $16.9(0.5)$ & 0.099 \\
\hline $\mathrm{IDL}-\mathrm{C}$ & $9.38(1.0)$ & $8.33(0.6)$ & $10.1(0.6)$ & 0.172 \\
\hline$\overline{L D L-C}$ & $100.1(6.2)$ & $92.9(3.6)$ & $102.7(4.3)$ & 0.227 \\
\hline TAG & $59.5^{a}(4.5)$ & $68.5^{\mathrm{a}, \mathrm{b}}(3.8)$ & $81.5^{b}(4.5)$ & 0.005 \\
\hline$\overline{L p(a)-C}$ & $10.7^{\mathrm{b}}(0.9)$ & $7.75^{\mathrm{a}}(0.5)$ & $6.71^{\mathrm{a}}(0.5)$ & $<0.001$ \\
\hline
\end{tabular}

${ }^{1}$ Differences determined using two-tailed t-test following Levene's test for equality of variances. Groups sharing a common superscript are not significantly different from each other using Tukey's studentized range test at a $5 \%$ procedure-wise error rate

Abbreviations: Body mass index: BMI, Body mass index z-score: BMIz, Waist circumference: WC, Total cholesterol: TC, High density lipoprotein cholesterol: HDL-C, Very low density lipoprotein cholesterol: VLDL-C, Intermediate density lipoprotein cholesterol: IDL-C, Low density lipoprotein cholesterol: LDL-C, Triglycerides: TAG, Lp(a)ccholesterol: Lp(a)-C

percentile subgroups ( $<$ 95th, 95.001-99th, $>$ 99th), differences among subgroups were statistically significant for concentrations of HDL-C, Lp(a)-C and TAG (Table 3).

Body fatness, assessed using either BMIz scores and WC, was significantly and negatively correlated with HDL-C and Lp(a)-C, and significantly but positively associated with TAG (Table 4). TC was positively associated with all concentrations of all lipoprotein subclasses and, among the subclasses, HDL-C was negatively associated with VLDL-C and TAG; and VLDL-C, IDL-C and LDL-C were intercorrelated. Lp(a)-
$\mathrm{C}$ was positively correlated with TC and HDL-C, and negatively correlated with VLDL-C and TAG.

Using multiple regression analysis, and following adjustments for child age, gender and pubertal stage, Lp(a)-C remained significantly and positively associated with $\mathrm{TC}$ and HDL-C, and remained significantly and negatively associated with VLDL-C and TAG (Table 5). Since obesity was highly correlated with $\mathrm{Lp}(\mathrm{a})-\mathrm{C}$, we included WC and BMIz (separately) as additional covariates. Associations of Lp(a)-C with TC, HDL-C, VLDL-C and TAG continued to be statistically significant after these adjustments for body fatness.

Table 4 Pearson correlation coefficients and significance ${ }^{1}$ among measure of obesity and plasma lipoprotein concentrations $(n=121)$

\begin{tabular}{|c|c|c|c|c|c|c|c|c|}
\hline & WC & TC & HDL-C & VLDL-C & IDL-C & LDL-C & TAG & Lp(a)-C \\
\hline BMlz & $0.801^{* * *}$ & -0.019 & $-0.348^{* * *}$ & $0.212^{*}$ & 0.134 & 0.090 & $0.357^{* * * *}$ & $-0.394^{* * *}$ \\
\hline$\overline{W C}$ & & -0.107 & $-0.476^{* * *}$ & $0.187^{*}$ & 0.104 & 0.050 & $0.332^{* * *}$ & $-0.337^{* * *}$ \\
\hline $\mathrm{TC}$ & & & $0.384^{* * *}$ & $0.310^{* * *}$ & $0.562^{* * *}$ & $0.934^{* * *}$ & $0.228^{*}$ & $0.219^{*}$ \\
\hline $\mathrm{HDL}-\mathrm{C}$ & & & & $-0.224^{*}$ & -0.117 & 0.051 & $-0.285^{* *}$ & $0.462^{* * *}$ \\
\hline VLDL-C & & & & & $0.803^{* * *}$ & $0.306^{* * *}$ & $0.869 * * *$ & $-0.254^{* *}$ \\
\hline IDL-C & & & & & & $0.574^{* * *}$ & $0.699^{* * *}$ & -0.155 \\
\hline LDL-C & & & & & & & $0.258^{* *}$ & 0.097 \\
\hline TAG & & & & & & & & $-0.283^{* *}$ \\
\hline
\end{tabular}

${ }^{1}$ Significance: ${ }^{*}$ for $p<0.05, * *$ for $p<0.01, * * *$ for $p<0.001$

Abbreviations: Body mass index: BMI, Body mass index z-score: BMIz, Waist circumference: WC, Total cholesterol: TC, High density lipoprotein cholesterol: HDL-C, Very low density lipoprotein cholesterol: VLDL-C, Intermediate density lipoprotein cholesterol: IDL-C, Low density lipoprotein cholesterol: LDL-C, Triglycerides: TAG, Lp(a) cholesterol: Lp(a)-C 
Table 5 Relationship of other plasma lipoproteins to Lp(a)-C concentrations $(n=121)$

\begin{tabular}{|c|c|c|c|c|c|c|c|}
\hline \multirow[b]{2}{*}{ Models } & \multirow[b]{2}{*}{ Other variables included in models } & \multicolumn{6}{|c|}{ Independent variables $^{1}$} \\
\hline & & TC & HDL-C & VLDL-C & IDL-C & LDL-C & TAG \\
\hline & & \multicolumn{6}{|c|}{ Standardized regression coefficient with $L p(a)$ as dependent variable ${ }^{2}$} \\
\hline I & None & $0.219^{*}$ & $0.462^{* * *}$ & $-0.254^{* *}$ & -0.155 & 0.097 & $-0.283^{* *}$ \\
\hline$\|$ & Covar & $0.244^{* *}$ & $0.541^{* * *}$ & $-0.276^{* *}$ & -0.166 & 0.114 & $-0.307^{* * *}$ \\
\hline$\underline{\text { III }}$ & Covar + BMlz & $0.235^{* *}$ & $0.446^{* * *}$ & $-0.196^{*}$ & -0.113 & 0.145 & $-0.187^{*}$ \\
\hline$\overline{I V}$ & Covar + WC & $0.227^{* *}$ & $0.450^{* * *}$ & $-0.200^{*}$ & -0.108 & 0.142 & $-0.190^{*}$ \\
\hline
\end{tabular}

${ }^{1}$ Regression analyses were performed separately for each independent lipoprotein variable. Coefficients are shown following adjustments for covariates (covar) including child sex, age, pubertal stage, group assignment and family socio-economic status

${ }^{2}$ Significance: ${ }^{*}$ for $p<0.05,{ }^{* *}$ for $p<0.01,{ }^{* *}$ for $p \leq 0.001$

Abbreviations: Body mass index: BMI, Body mass index z-score: BMIz, Waist circumference: WC, Total cholesterol: TC, High density lipoprotein cholesterol: HDL-C, Very low density lipoprotein cholesterol: VLDL-C, Intermediate density lipoprotein cholesterol: IDL-C, Low density lipoprotein cholesterol: LDL-C, Triglycerides: TAG, Lp(a) cholesterol: Lp(a)-C

Since Lp(a)-C appeared to be consistently and more strongly related to HDL-C than to the other lipoprotein subclasses (Table 5), a final analysis was performed to determine if the association between $\mathrm{Lp}(\mathrm{a})-\mathrm{C}$ and HDL$\mathrm{C}$ was accounted for by other plasma lipoproteins since inter-correlations were observed among the lipoprotein subclasses (Table 5). The strong relationship between HDL-C and Lp(a)-C was not explained by its association with other lipoprotein subclasses since, after adjusting for each subclass in a separate regression model, $\mathrm{Lp}(\mathrm{a})$ $\mathrm{C}$ remained highly related to HDL-C whereas associations of $\mathrm{Lp}(\mathrm{a})-\mathrm{C}$ with other lipoprotein subclasses were no longer statistically significant (Table 6).

Finally, we evaluated the frequency with which children in our cohort were simultaneously "at risk" of CVD using the cutoffs provided in Table 1 . None of the children in the "at risk" categories using cutoffs for HDL-C, VLDL-C, IDL-C or TAG were in the "at risk" category for $\mathrm{Lp}(\mathrm{a})$ (data not shown). Of the children in the "at risk" categories using the cutoffs for TC or LDL$C$, only $1 / 3$ were in the "at risk" category for $L p(a)$.

\section{Discussion}

To our knowledge this is one of the first studies to report a strong positive association between $\mathrm{Lp}(\mathrm{a})-\mathrm{C}$ and HDL-C in overweight and obese African American children. Positive correlations between Lp(a)-C and HDL-C concentrations have been reported previously in premenopausal African American women [16], and our results show that this relationship can be observed prior to adulthood. Although, others have reported no association between $\mathrm{Lp}(\mathrm{a})-\mathrm{C}$ and HDL-C in normal weight girls [25], our results expand on the current literature by showing that the strong positive association between $\mathrm{Lp}$ (a)-C and HDL-C in overweight and obese African American children included in our study. This association remained following adjustments for body fatness and following adjustments for other plasma lipoprotein subclasses.

The strong association of $\mathrm{Lp}(\mathrm{a})-\mathrm{C}$ with HDL-C observed in our study suggests that increasing $\mathrm{Lp}(\mathrm{a})-\mathrm{C}$ concentrations may not be reliably used as a marker of CAD risk in this population. Instead, our results agree

Table 6 Relationship of plasma concentrations of Lp(a)-C to HDL-C before and after adjustments for other plasma lipoproteins $(n=121)$

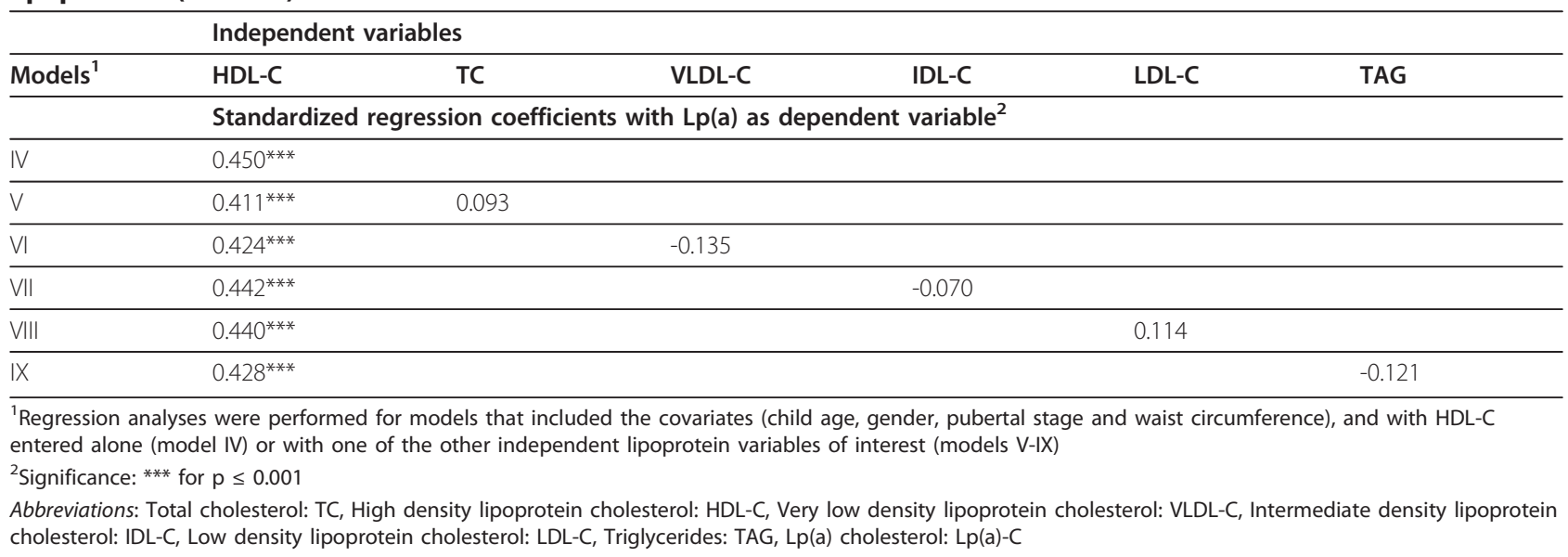


with those of others [17] who observed that a therapeutic lifestyle modification program administered to obese African Americans aged 12-75 years caused levels of Lp (a) and HDL-C to increase and caused LDL-C, TC, TG and BMI to decrease. Those authors reasoned that, since previous studies with therapeutic life style change have shown a favorable impact on cardio vascular health, their results, like ours, did not support the established understanding that elevations in $\mathrm{Lp}$ (a) would be atherogenic and harmful. Thus, our results in overweight and obese African American children are in line with the suggestion, made by others [6], that increasing plasma concentration of $L p(a)$ is not an independent risk factor for CVD in African American adults.

$\mathrm{Lp}(\mathrm{a})$ is known to consist of an LDL-like particle covalently linked via an apo-B100 molecule to apolipoprotein (a), and these particles are variable in its size and cholesterol content. $\mathrm{Lp}(\mathrm{a})$ and LDL have been reported to be positively correlated in African American females $[16,25]$ and in Taiwanese boys [15] and white girls [11]. Relationships were reported not to be significant, however, in Taiwanese girls [15], white boys, black boys and black girls [11]. In our population, $\mathrm{Lp}(\mathrm{a})-\mathrm{C}$ and LDL-C concentrations also were not found to be significantly associated even after adjusting for child characteristics including degree of obesity. This lack of association is unlikely to be due to the analytical procedure used since $\mathrm{Lp}(\mathrm{a})-\mathrm{C}$, measured using the VAP-C technique as we have done, has been reported to be highly correlated to Lp(a) mass measured using ELISA [23].

In our cohort of overweight African American children, $L p(a)$ was significantly and negatively associated with TAG concentrations prior to an following adjustment for obesity. Others have reported also a negative association between LP(a) and TAG in American Americans aged 12-75 [17], African American women [16], Turkish men and women [10], and Taiwanese girls [15]. Although our results agree with those of others, the association was no longer significant following adjustment for HDL-C. This suggests that HDL-C accounts for the association between $\mathrm{Lp}(\mathrm{a})$ and TAG observed by us and others.

Others have previously reported that African American children and adolescents have higher levels of $\mathrm{Lp}(\mathrm{a})$ and HDL than their non-Hispanic white peers $[11,25,26]$. In our cohort, nearly $25 \%$ of children had Lp (a)-C concentrations that placed them in the "at risk" category whereas only $12 \%$ had HDL-C concentrations that placed them in the "at risk" category. Of interest, is our observation that, of the 15 individual children in our cohort with low "at risk" HDL-C, none had "at risk" $\mathrm{Lp}(\mathrm{a})-\mathrm{C}$ concentrations. Thus, the strong positive association we observed between $\mathrm{Lp}(\mathrm{a})-\mathrm{C}$ and HDL-C suggests it is unlikely that both of these factors would serve as a reliable marker for risk of CVD in overweight and obese African American children.

In our population, $\mathrm{Lp}(\mathrm{a})-\mathrm{C}$ concentrations were negatively related to body fatness assessed using either BMIz or WC, and children between the 85th and 95th BMI percentile had significantly higher $\mathrm{Lp}(\mathrm{a})$-C concentrations than children with BMIs above the 95th percentile. Previously, $\mathrm{Lp}(\mathrm{a})-\mathrm{C}$ concentrations were reported to be negatively correlated with body weight and waist-to-hip ratio in African American women [16], although no association was found between $\mathrm{Lp}(\mathrm{a})$ levels and obesity in non-African American children or adults [15,27]. Because $L p(a)-C$ was correlated with obesity in our population, BMIz or WC were included as covariates in our multiple regression models. Our results show, however, that the positive association between $\mathrm{Lp}(\mathrm{a})-\mathrm{C}$ and HDL-C was independent of the degree of obesity in these children.

This pilot analysis includes restriction to low-income, inner-city, African American children and exclusion of children with BMI's less than the 85th percentile when matched for age and gender. These limitations preclude extrapolation to the wider population of children of different races, ages and socioeconomic backgrounds, and comparisons with lower BMI children. This is a crosssectional analysis of data, precluding a cause and effect relationship. Lipoprotein subclasses were analyzed using the VAP-cholesterol technique, precluding extrapolation to results based on other analytical techniques that quantify numbers or size of particles or associated protein.

\section{Conclusions}

Lp(a)-C was positively associated with HDL-C in overweight and obese African American children and this association remained following adjustments for other lipoprotein subclasses and degree of obesity. Based on our study, we propose that $\mathrm{Lp}(\mathrm{a})$ is not associated with lipoprotein recognized as markers of increased risk of CVD in overweight and obese African American children. Further studies will be needed to explain the basis for the association of $L p(a)-C$ and HDL-C in these children.

\footnotetext{
Abbreviations

Lp(a): Lipoprotein (a); Lp(a)-C: Lp(a) cholesterol; LDL-C: Low density lipoprotein cholesterol; HDL-C: High density lipoprotein cholesterol; IDL-C: Intermediate density lipoprotein cholesterol; VLDL-C: Very low density lipoprotein cholesterol;TAG: Triglycerides; TC: Total cholesterol; BMI: Body mass index; BMlz: Body mass index z-score; WC: Waist circumference; CVD: Cardiovascular disease.
}

\section{Acknowledgements}

Essential funding was provided by USDA CSREES grants 2004-35214-14254 and 2005-35215-15046, the Agriculture Experiment Station and the YMCA. 


\section{Author details}

'Dr Robert C and Veronica Atkins Center for Weight and Health, University of California, Berkeley, CA 94720-3104, USA. 'Department of Nutritional Sciences and Toxicology, University of California, Berkeley, CA 94720-3104, USA. ${ }^{3}$ Children's Hospital Oakland Research Institute, Oakland, CA 94609, USA.

\section{Authors' contributions}

Contributor's list: Dr.S contributed in statistical analysis, prepared the manuscript and submission. Ms. Merchant provided analytical support and expertise in interpretation of data. Prof.F was the principal investigator of the study. She supervised the design and execution of the study and manuscript. All authors read and approved the final manuscript.

\section{Competing interests}

The authors declare that they have no competing interests.

Received: 3 December 2011 Accepted: 27 January 2012 Published: 27 January 2012

\section{References}

1. Berg K: A new serum type system in man-the Lp system. Acta Pathol Microbiol Scand 1963, 59:369-382.

2. Utermann G, Weber W: Protein composition of $L p(a)$ lipoprotein from human plasma. FEBS Lett 1983, 154(2):357-361.

3. Djurovic S, Berg K: Epidemiology of Lp(a) lipoprotein: its role in atherosclerotic/thrombotic disease. Clin Genet 1997, 52(5):281-292.

4. Brown SA, et al: The relation of lipoprotein[a] concentrations and apolipoprotein[a] phenotypes with asymptomatic atherosclerosis in subjects of the Atherosclerosis Risk in Communities (ARIC) Study. Arterioscler Thromb 1993, 13(11):1558-1566.

5. Schreiner PJ: Lipoprotein(a) as a risk factor for preclinical atherosclerotic disease in a biracial cohort: the Atherosclerosis Risk in Communities (ARIC) Study. Chem Phys Lipids 1994, 67-68:405-410.

6. Moliterno DJ, et al: No association between plasma lipoprotein(a) concentrations and the presence or absence of coronary atherosclerosis in African-Americans. Arterioscler Thromb Vasc Biol 1995, 15(7):850-855.

7. Dangas $G$, et al: Correlation of serum lipoprotein(a) with the angiographic and clinical presentation of coronary artery disease. Am J Cardiol 1999, 83(4):583-585, A7.

8. Marcovina SM, et al: Lipoprotein[a] concentrations and apolipoprotein[a] phenotypes in Caucasians and African Americans. The CARDIA study. Arterioscler Thromb 1993, 13(7):1037-1045.

9. Pearson TA, et al: AHA Guidelines for Primary Prevention of Cardiovascular Disease and Stroke: 2002 Update: Consensus Panel Guide to Comprehensive Risk Reduction for Adult Patients Without Coronary or Other Atherosclerotic Vascular Diseases. American Heart Association Science Advisory and Coordinating Committee. Circulation 2002, 106(3):388-391.

10. Onat A, et al: Lipoprotein (a) in a population-based study: more significant in Turkish women than men? Anadolu Kardiyol Derg 2005, 5(4):271-277.

11. Heyden S, et al: Raised Lipoprotein(a) in Hypercholesterolemic BlackStudents Compared to Age-Matched Whites in North and SouthCarolina. Int J Epidemiol 1994, 23(2):301-306

12. Dahlen GH, et al: Association of levels of lipoprotein $L p(a)$, plasma lipids, and other lipoproteins with coronary artery disease documented by angiography. Circulation 1986, 74(4):758-765.

13. Howard BV, et al: Concentrations of $L p(a)$ in black and white young adults: relations to risk factors for cardiovascular disease. Ann Epidemiol 1994, 4(5):341-350.

14. Cunningham TE, Sayers SM, Singh GR: Lipoprotein(a) identifies cardiovascular risk in childhood: the Australian aboriginal birth cohort study. J Paediatr Child Health 2011, 47(5):257-261.

15. Chu NF, et al: Lipoprotein profiles, not anthropometric measures, correlate with serum lipoprotein(a) values in children: the Taipei children heart study. Eur J Epidemiol 2000, 16(1):5-12.

16. Giger JN, et al: Genetic predictors of coronary heart disease risk factors in premenopausal African-American women. Ethn Dis 2005, 15(2):221-232.
17. Randall OS, et al: Response of lipoprotein(a) levels to therapeutic lifestyle change in obese African-Americans. Atherosclerosis 2004, 172(1):155-160.

18. Berenson GS, et al: Association between multiple cardiovascular risk factors and atherosclerosis in children and young adults. The Bogalusa Heart Study. N Engl J Med 1998, 338(23):1650-1656.

19. Hirschler $\mathrm{V}$, et al: Waist circumference identifies primary school children with metabolic syndrome abnormalities. Diabetes Technol Ther 2007, 9(2):149-157.

20. van Vliet $\mathrm{M}$, et al: Cardiometabolic risk variables in overweight and obese children: a worldwide comparison. Cardiovasc Diabetol 2011, 10(1):106

21. Ritchie LD, et al: Taking action together: a YMCA-based protocol to prevent type-2 diabetes in high-BMI inner-city African American children. Trials 2010, 11:60.

22. Kulkarni KR, et al: Identification and cholesterol quantification of low density lipoprotein subclasses in young adults by VAP-II methodology. J Lipid Res 1995, 36(11):2291-2302.

23. Kulkarni KR, et al: Quantification of cholesterol in all lipoprotein classes by the VAP-II method. J Lipid Res 1994, 35(1):159-168.

24. Raman A, et al: Accuracy of self-assessed Tanner staging against hormonal assessment of sexual maturation in overweight AfricanAmerican children. J Ped Endocrin Metab 2009, 22:609-622.

25. Kimm SY, et al: Correlates of lipoprotein(a) levels in a biracial cohort of young girls: the NHLBI Growth and Health Study. J Pediatr 1999, 135(2 Pt 1):169-176.

26. Herd SL, et al: Body fat, fat distribution and serum lipids, lipoproteins and apolipoproteins in African-American and Caucasian-American prepubertal children. Int J Obes Relat Metab Disord 2001, 25(2):198-204.

27. Corsetti JP, et al: Effect of weight loss on serum lipoprotein(a) concentrations in an obese population. Clin Chem 1991, 37(7):1191-1195.

doi:10.1186/1475-2840-11-10

Cite this article as: Sharma et al:: $\mathrm{Lp}(\mathrm{a})$-cholesterol is associated with HDL-cholesterol in overweight and obese African American children and is not an independent risk factor for CVD. Cardiovascular Diabetology 2012 11:10

\section{Submit your next manuscript to BioMed Central and take full advantage of:}

- Convenient online submission

- Thorough peer review

- No space constraints or color figure charges

- Immediate publication on acceptance

- Inclusion in PubMed, CAS, Scopus and Google Scholar

- Research which is freely available for redistribution

Submit your manuscript at www.biomedcentral.com/submit
Biomed Central 